\title{
A Simple Noninvasive Score Predicts Disease Activity and Deep Remission in Ulcerative Colitis
}

\author{
Amr Shaaban Hanafy ${ }^{a}$ Mohamed Hesham Monir ${ }^{a}$ Hany Abdel Malak ${ }^{b}$ \\ Mohamed Desoky Aiad ${ }^{\mathrm{C}}$ \\ a Internal Medicine Department, Hepatogastroenterology and Endoscopy Division, Zagazig University, \\ Zagazig, Egypt; ${ }^{b}$ Clinical Pathology, Zagazig University, Zagazig, Egypt; ' Internal Medicine Department, \\ Hepatogastroenterology Division, Zagazig University, Al Ahrar Hospital, Zagazig, Egypt
}

\section{Keywords}

Noninvasive score $\cdot$ Prediction · Disease activity · Deep remission - Ulcerative colitis

\begin{abstract}
Background: There is a need to use noninvasive markers in refining the management of ulcerative colitis to reduce the number of unnecessary colonoscopies, which facilitates the follow-up of activity and the response to treatment. Aim: Postulation of a sensitive, specific, simple and noninvasive score to monitor disease activity in ulcerative colitis. Methods: A case-control study was conducted: 168 patients with ulcerative colitis, 40 healthy individuals, and 60 patients for validation. Patients were divided into new diagnosis $(n=50)$, clinical remission $(n=60)$, and relapse $(n=58)$. The main outcome measures if the score correlates with clinical, endoscopic and histopathological characteristics and if it correlates with deep remission. Results: A scoring system was established composed of lactoferrin at a cutoff of $148.5 \mu \mathrm{g} / \mathrm{mL}$, neutrophil lymphocyte ratio at a cutoff of 2.35, erythrocyte sedimentation rate at the first hour at a cutoff of $29.5 \mathrm{~mm} / \mathrm{h}$, C-reactive protein at a cutoff of $3.85 \mathrm{mg} / \mathrm{L}$, mean platelet volume at a cutoff of $8.8 \mathrm{fL}$, fecal white blood cells at a cutoff of
\end{abstract}

9 cells/HPF, and fecal red blood cells at a cutoff of 6 cells/HPF. A score $\geq 5$ can detect $94 \%$ of cases of UC as determined by the receiver operating characteristic curve with a sensitivity of $94 \%$ and a specificity $100 \%$, AUC 0.92, SE 0.05, $p=0.001$, $95 \% \mathrm{Cl} 0.82-1.1$. In the validation group, it identified severely affected patients with a sensitivity of $95 \%$ and a specificity of $85.7 \%$. Conclusions: This easily applied and reproducible noninvasive activity score showed high performance in predicting disease activity and deep remission in ulcerative colitis.

(c) 2018 S. Karger AG, Basel

\section{Introduction}

Ulcerative colitis (UC) is one of the major forms of inflammatory bowel disease (IBD), which additionally includes Crohn's disease and indeterminate colitis, all characterized by idiopathic chronic inflammation of the gastrointestinal tract [1].

The use of noninvasive markers in modulating the management of US reduces the number of colonoscopies with possible economic effects and facilitates the follow-up of activity and the response to treatment.

\section{KARGER}

(c) 2018 S. Karger AG, Basel

E-Mail karger@karger.com

www.karger.com/iid
Amr Shaaban Hanafy

40-Mostafa Fouad St.

Sharkia, Zagazig 44519 (Egypt)

E-MailDr_amr_hanafy@yahoo.com 
They should be sensitive, specific, simple, and time- and cost-saving.

Lactoferrin (LF) is an iron-binding glycoprotein and a major component of the neutrophilic secondary granules. Leukocyte infiltration of the gastrointestinal mucosa increases fecal LF concentration. LF was found to be a sensitive and specific marker in measuring the activity of IBD and valuable in discriminating inflammatory from noninflammatory bowel states $[2,3]$.

Complete blood count provides efficient, easy, and inexpensive markers of inflammation, such as mean platelet volume (MPV) and neutrophil lymphocyte ratio (NLR). Platelet count is increased in patients with IBD, mainly reticulated platelets due to the thrombogenic nature of UC [4]. MPV represents an indicator of platelet activation and is positively correlated with white blood cell (WBC) count, erythrocyte sedimentation rate (ESR), and C-reactive protein (CRP); however, it is inversely correlated with IBD activity [5].

NLR is a simple, inexpensive marker of inflammation that had been linked with several inflammatory and neoplastic diseases including colorectal cancer, gastric cancer, hepatocellular carcinoma, and pancreatic cancer. It reflects the magnitude of disease activity and is a useful marker for estimating intestinal inflammation [6].

The aim of the current study is to facilitate monitoring disease activity, to follow up the response to treatment, and to predict relapse in UC by noninvasive markers and correlate these markers to both clinical and endoscopic severity of the disease.

\section{Methods}

\section{Patient Selection}

A case-control study was conducted in the IBD Clinic and Endoscopy Unit of the Internal Medicine Department, Zagazig University, Egypt, which is a tertiary referral center, in the period from July 2014 to August 2016.

390 patients were evaluated during this period; 222 patients were excluded as shown in Figure 1, i.e., 168 patients (100 males and 68 females) fulfilled the inclusion criteria and were enrolled in study after being diagnosed with UC.

The patients were further subdivided into 3 groups: treatmentnaïve with a new diagnosis $(n=50)$, patients in remission $(n=60)$, and patients with relapse $(n=58)$.

Patients were included if the diagnosis of UC was documented using the clinical picture, laboratory, colonoscopic and histological findings. Clinical severity of active UC was evaluated by Truelove and Witts' classification [7].

The exclusion criteria included patients who refused to participate in the study or to undergo colonoscopy, other forms of IBD such as Crohn's disease, acute infectious colitis, and a history of chronic NSAID intake or exposure to radiation.
Informed consent was obtained from all the participants before enrollment in the study, which was approved by the ethics committee of Zagazig University Hospital.

Community-Based Controls

40 healthy age- and sex-matched controls were recruited from the outpatient clinic. They were investigated by the same approach for comparison.

\section{Validation}

The aim was to clinically assess the predictive power of the scoring model through evaluation in an age-matched group composed of 60 patients with clinical manifestations suggesting the diagnosis of UC. The methods used were similar to those of the study patients. All the patients had been exposed to colonoscopy.

\section{Clinical Evaluation}

All the patients were subjected to full history taking and thorough physical examination.

\section{Laboratory Analysis}

- Fecal LF: fresh or frozen stool samples assayed by the Human Lactoferrin ELISA Kit (Glory Science Co., Ltd., USA). Reference value in the stool is less than $7.25 \mu \mathrm{g} / \mathrm{mL}$.

- A single, fresh stool specimen was examined within an hour of collection for the presence of visible blood and mucus; the numbers of red blood cells (RBCs) and WBCs were calculated and expressed as average numbers under the following categories: $0,1-10,11-20,21-50$, and $>50$ cells/HPF.

- Complete blood counts were done on the samples within $6 \mathrm{~h}$ after phlebotomy. The NLR was calculated by dividing the mean neutrophil count by the mean lymphocyte count with a cutoff value $>2$. The MPV was detected by Sysmex from ethylenediaminetetraacetic acid blood samples, which were rapidly processed within $1 \mathrm{~h}$ in order to minimize platelet swelling with a reference range of 7.8-11.0 fL.

- CRP was measured using a nephelometric method. In apparently healthy persons, blood CRP levels are below $5 \mathrm{mg} / \mathrm{L}$.

- ESR at the 1st hour (ESR1) and 2nd hour (ESR2), liver function tests, kidney function tests.

- Prothrombin time, partial thromboplastin time, and international normalized ratio.

- Imaging as abdominal X-ray in severe UC and abdominal ultrasonography.

\section{Colonoscopy}

Contraindications to colonoscopy preparation included fulminant UC, toxic megacolon, perforation, intestinal obstruction, and hemodynamic instability. The endoscopic severity of UC was assessed using the Mayo Clinic subscore [8]. For the initial diagnosis of UC, multiple biopsies (at least 2) were taken from 5 sites around the colon including the rectum and ileum.

\section{Statistical Analysis}

Data were analyzed using PASW Statistics 18. The sample size was calculated from the equation $=(z \text { score })^{2} \times \mathrm{SD} \times(1-\mathrm{SD}) /$ $(\text { margin of error })^{2}$; thus, $(1.96)^{2} \times 0.5 \times(0.5) /(0.05)^{2}=384$ participants were needed. However, 390 patients were collected in the predefined period of the study as shown in Figure 1. 


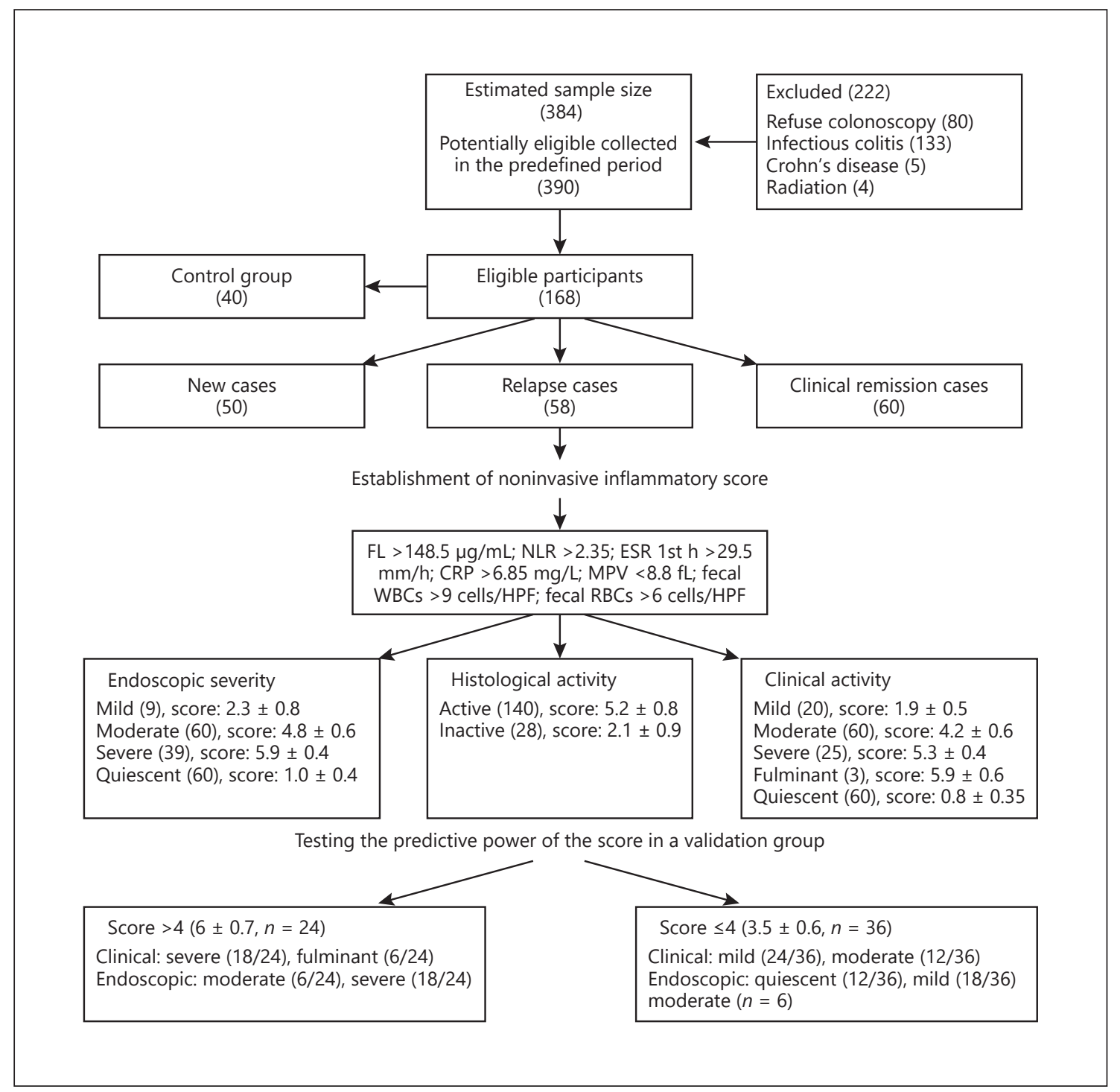

Fig. 1. Flow diagram of the study and validation groups.

Continuous variables were summarized as mean \pm standard deviation and standard error (SE) when appropriate. The $\chi^{2}$ test, Student $t$ test, and analysis of variance were appropriately used. The potential risk factors associated with activity in UC were correlated using Spearman's rank correlation. Correlations were significant if $p<0.05$. Regression analysis, estimation of odds ratios, and corresponding $\beta$ coefficients were assessed. A receiver operating characteristic (ROC) analysis was carried out to detect the cutoff value of each variable and examine the diagnostic utility of the constructed scoring model.

A scoring system was postulated composed of the highly correlated variables. Points were assigned to each variable based on the magnitude of its regression coefficient; the one with the smallest $\beta$ coefficient was given 1 point and the others were given points according to their strength when compared to the smallest. The summed points were grouped into predefined categories for the risk of UC: no risk, low (<15\%), intermediate (15-49\%), high (50$79 \%)$, and very high (>80\%) [9].

\section{Results}

The study included 168 patients with UC who were subdivided into 3 groups: newly diagnosed $(n=50)$ with a mean age of $33 \pm 8$ years, relapse group $(n=58)$ with a mean age of $34.9 \pm 11$ years, and remission group $(n=60)$ with a mean age of $33 \pm 12$ years $(p=0.8)$. The mean values of the body mass index for the 3 groups were $25 \pm 3.7$, 


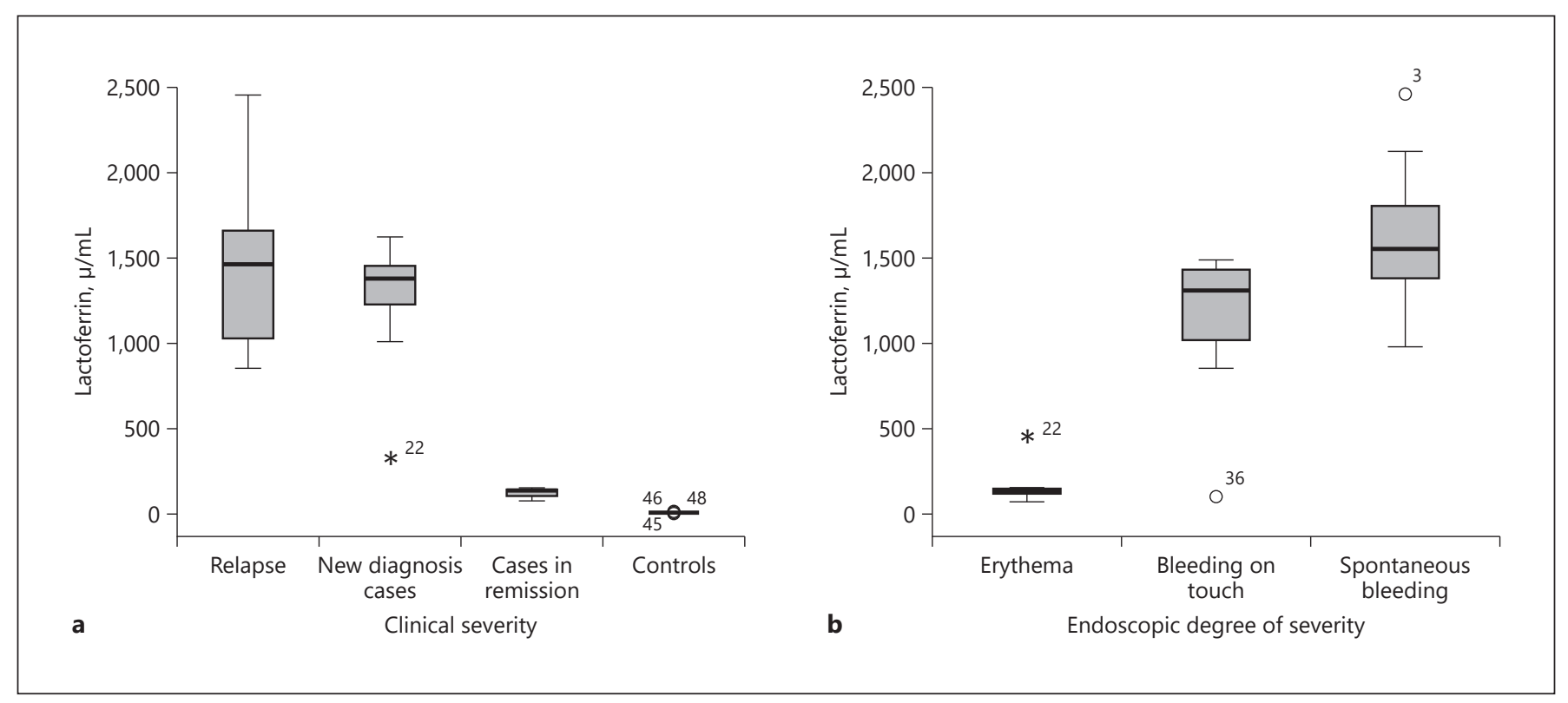

Fig. 2. a Level of fecal lactoferrin according to clinical severity in the studied groups. b Level of fecal lactoferrin according to the endoscopic degree of severity.

Table 1. Frequency of symptoms among ulcerative colitis patients

\begin{tabular}{|c|c|c|c|c|c|c|}
\hline \multirow{3}{*}{$\begin{array}{l}\text { Clinical } \\
\text { characters }\end{array}$} & \multicolumn{4}{|c|}{ Studied groups } & \multicolumn{2}{|c|}{ Remission } \\
\hline & & isease & & nosis & $n$ & $\%$ \\
\hline & $n$ & $\%$ & $n$ & $\%$ & & \\
\hline
\end{tabular}

\begin{tabular}{lrrrrrr}
\hline Bowel movement & & & & & & \\
$\quad<4$ & 13 & 22 & 4 & 8 & 60 & 100 \\
$\quad 4-6$ & 28 & 48 & 32 & 64 & & \\
$\quad 6$ & 17 & 30 & 14 & 28 & & \\
Bleeding & 58 & 100 & 50 & 100 & - & - \\
Pain & 58 & 100 & 50 & 100 & - & - \\
Tenesmus & 44 & 76 & 40 & 80 & - & - \\
Urgency & 34 & 59 & 38 & 76 & - & - \\
Clinical severity & & & & & & \\
$\quad$ Mild & 12 & 20.7 & 8 & 16 & & \\
$\quad$ Moderate & 32 & 55.2 & 28 & 56 & & \\
$\quad$ Sever & 14 & 24.1 & 11 & 22 & & \\
$\quad$ Fulminant & 0 & 0 & 3 & 6 & & \\
$\quad$ Quiescent & & & & & 60 & 100 \\
\hline
\end{tabular}

$26.4 \pm 4.4$, and $25.9 \pm 3.7$, respectively $(p=0.7)$. The total number of nonsmokers in the 3 groups was 140 patients (83.3\%), 20 patients (12\%) were smokers, while 8 patients (4.7\%) were ex-smokers $(p=0.8)$.

The frequency of symptoms among the studied patients is shown in Table 1. Fecal LF mean values were sig- nificantly higher in the newly diagnosed and relapse groups when compared to the remission group $(1,291 \pm$ 285.6, $1,458 \pm 479.5$, and $121 \pm 26.7 \mu \mathrm{g} / \mathrm{mL}$, respectively, $p=0.000)$ (Fig. 2a; Table 2).

Mean hemoglobin concentration was significantly lower in the newly diagnosed and relapse groups when compared to the remission group $(10.3 \pm 2,10.3 \pm 1.6$, and 12.5 $\pm 1.6 \mathrm{~g} / \mathrm{dL}$, respectively, $p=0.002)$. Mean corpuscular volume showed an insignificant statistical difference $(p=0.7)$.

WBC count showed an insignificant statistical difference among the 3 groups ( $p=0.07$ ); however, NLR mean values were significantly higher in the newly diagnosed and relapse groups $(4.8 \pm 2.9,4.4 \pm 1.5)$ when compared to the remission group $(1.8 \pm 0.68)(p=0.000)$. Platelet count showed an insignificant statistical difference $(p=$ 0.06 ). Meanwhile, MPV mean values were significantly lower in the newly diagnosed and relapse groups (7.7 \pm $0.97,8.2 \pm 1.69 \mathrm{fL})$ when compared to the remission group $(9.3 \pm 1 \mathrm{fL})(p=0.008)$.

ESR1, ESR2 and CRP were significantly higher in the newly diagnosed and relapse groups when compared to the remission group $(p=0.001)$. Serum albumin was significantly lower in the newly diagnosed and relapse groups $(3 \pm 0.78,3.6 \pm 1 \mathrm{~g} / \mathrm{dL})$ when compared to the remission group ( $4.2 \pm 0.5 \mathrm{~g} / \mathrm{dL} ; p=0.002)$ (Table 2$)$.

The control group included 40 subjects with a mean age of $35.2 \pm 2.5$ years, mean fecal LF $5.4 \pm 1.3 \mu \mathrm{g} / \mathrm{mL}$, 
Table 2. Laboratory findings of ulcerative colitis patients

\begin{tabular}{|c|c|c|c|c|c|}
\hline & \multicolumn{3}{|l|}{ Studied groups } & \multirow[t]{3}{*}{$F$} & \multirow[t]{3}{*}{$p$} \\
\hline & \multicolumn{2}{|l|}{ active disease } & \multirow[t]{2}{*}{ remission } & & \\
\hline & relapse & new diagnosis & & & \\
\hline Lactoferrin, $\mu \mathrm{g} / \mathrm{mL}$ & $1,458.6 \pm 479.5$ & $1,291 \pm 285.6$ & $121 \pm 26.7$ & 71.4 & 0.0 \\
\hline $\mathrm{HB}, \mathrm{g} / \mathrm{dL}$ & $10.3 \pm 1.6$ & $10.3 \pm 2$ & $12.5 \pm 1.6$ & 7.4 & 0.002 \\
\hline $\mathrm{MCV}, \mathrm{fL}$ & $83.3 \pm 13$ & $81.3 \pm 10.8$ & $84.4 \pm 8.9$ & 0.29 & 0.7 \\
\hline $\mathrm{WBC}, 10^{3} / \mu \mathrm{L}$ & $11.6 \pm 6$ & $10.8 \pm 5$ & $7.7 \pm 2.1$ & 2.7 & 0.07 \\
\hline NLR & $4.4 \pm 1.5$ & $4.8 \pm 2.9$ & $1.8 \pm 0.68$ & 10 & 0.0 \\
\hline $\mathrm{PLT}, 10^{3} / \mu \mathrm{L}$ & $341 \pm 128$ & $406 \pm 136$ & $295 \pm 89$ & 3 & 0.06 \\
\hline MPV, $\mathrm{fL}$ & $8.2 \pm 1.69$ & $7.7 \pm 0.97$ & $9.3 \pm 1$ & 5.4 & 0.008 \\
\hline $\mathrm{ESR} 1, \mathrm{~mm} / \mathrm{h}$ & $47 \pm 15$ & $37 \pm 13$ & $18 \pm 10$ & 18.5 & 0.000 \\
\hline $\mathrm{ESR} 2, \mathrm{~mm} / \mathrm{h}$ & $89 \pm 20$ & $63 \pm 21$ & $20 \pm 5$ & 18.1 & 0.000 \\
\hline CRP, mg/L & $25.5 \pm 15$ & $24 \pm 23.8$ & $3.7 \pm 1.9$ & 7.8 & 0.001 \\
\hline AST, IU/L & $20 \pm 8$ & $18 \pm 7$ & $21 \pm 8$ & 0.3 & 0.7 \\
\hline ALT, IU/L & $23 \pm 9$ & $19 \pm 7$ & $19 \pm 5$ & 1.6 & 0.2 \\
\hline Albumin, g/dL & $3.6 \pm 1$ & $3 \pm 0.78$ & $4.2 \pm 0.5$ & 7.3 & 0.002 \\
\hline Bilirubin, mg/dL & $0.6 \pm 0.27$ & $0.6 \pm 0.25$ & $0.7 \pm 0.2$ & 0.15 & 0.8 \\
\hline
\end{tabular}

$F$ value, variance of the group means in ANOVA; MCV, mean corpuscular volume.

Table 3. Laboratory findings of patients with histologically active and inactive ulcerative colitis

\begin{tabular}{lcccc}
\hline Markers of activity & Histological activity & \multirow{2}{*}{$p$} & $p$ \\
\cline { 2 - 3 } & active $(n=140)$ & inactive $(n=28)$ & & \\
\hline Fecal lactoferrin, $\mu \mathrm{g} / \mathrm{mL}$ & $1,124.8 \pm 618$ & $117 \pm 34$ & 18.2 & 0.000 \\
WBC, $10^{3} / \mu \mathrm{L}$ & $10.6 \pm 5.1$ & $7.3 \pm 1.8$ & 2.7 & 0.016 \\
NLR & $4.4 \pm 2.4$ & $2 \pm 0.52$ & 4.8 & 0.03 \\
Platelet count, $10^{3} / \mu \mathrm{L}$ & $351 \pm 129$ & $327 \pm 110$ & 0.22 & 0.64 \\
MPV, fL & $8.2 \pm 1.3$ & $9.6 \pm 1$ & 7 & 0.01 \\
ESR1, mm/h & $36.7 \pm 17.6$ & $22 \pm 10$ & 4.4 & 0.04 \\
CRP, mg/L & $20.7 \pm 19.4$ & $3.2 \pm 1$ & 5.5 & 0.02 \\
Albumin, g/dL & $3.5 \pm 0.97$ & $4 \pm 0.49$ & 2.1 & 0.14 \\
\hline
\end{tabular}

CRP $2.9 \pm 1.3 \mu \mathrm{g} / \mathrm{L}, \mathrm{ESR} 7.4 \pm 0.5, \mathrm{MPV} 10.5 \pm 0.9 \mathrm{fL}$, and NLR $1.2 \pm 0.3$.

The relation between markers of activity of UC and histological activity in biopsies retrieved by colonoscopy in all the patients $(n=168)$ are shown in Table 3.28 patients showed inactive disease and all belong to the remission group. Histological activity was evident in 140 patients (83.3\%), i.e., 50 new cases, 58 relapse cases, and 32 patients in the clinical remission group; the latter group showed cryptitis without abscess or ulcer formation despite clinical and endoscopic remission.
Fecal LF, WBC count, NLR, ESR1, and CRP were significantly higher and MPV was significantly lower in the histologically active UC patients when compared to the histologically inactive patients, and this magnifies the role of the noninvasive markers in discriminating deep remission from clinical and endoscopic remissions.

Regarding endoscopic severity of UC, the Mayo Clinic subscore classifies UC into (0) quiescent or normal, (1) mild, (2) moderate, and (3) severe. Fecal LF, NLR, ESR1, and CRP were significantly higher in the UC patients with moderate and severe endoscopic changes. MPV and serum albumin were also significantly lower in these groups 
Table 4. Correlation between laboratory findings and endoscopic severity of UC patients

\begin{tabular}{|c|c|c|c|c|c|c|}
\hline \multirow{2}{*}{$\begin{array}{l}\text { Markers of ulcerative } \\
\text { colitis }\end{array}$} & \multirow{2}{*}{$\begin{array}{l}\text { Quiescent } \\
(n=60)\end{array}$} & \multicolumn{3}{|c|}{ Endoscopic finding } & \multirow[t]{2}{*}{$F$} & \multirow[t]{2}{*}{$p$} \\
\hline & & mild $(n=9)$ & moderate $(n=60)$ & severe $(n=39)$ & & \\
\hline WBC & $6.8 \pm 1.9$ & $7.6 \pm 2.7$ & $11 \pm 6.8$ & $11.4 \pm 3$ & 2.6 & 0.08 \\
\hline NLR & $1.7 \pm 0.4$ & $1.9 \pm 0.6$ & $4 \pm 2.1$ & $5.2 \pm 2.5$ & 9.8 & 0.000 \\
\hline PLT & $210 \pm 67$ & $295 \pm 94$ & $373 \pm 129$ & $379 \pm 138$ & 1.9 & 0.16 \\
\hline CRP & $5.3 \pm 1.6$ & $7.3 \pm 2.2$ & $24.4 \pm 19$ & $25.5 \pm 21$ & 7.5 & 0.002 \\
\hline Albumin & $4 \pm 0.5$ & $3.8 \pm 0.6$ & $3.5 \pm 0.9$ & $3.1 \pm 1$ & 3.6 & 0.03 \\
\hline
\end{tabular}

$F$ value, variance of the group means in ANOVA.

Table 5. Endoscopic activity and site of lesions in the studied subgroups

Finding

\begin{tabular}{lll}
\multicolumn{1}{l}{ Studied groups } & & \\
$\begin{array}{l}\text { relapse } \\
(n=58)\end{array}$ & $\begin{array}{l}\text { new diagnosis } \\
(n=50)\end{array}$ & $\begin{array}{l}\text { remission } \\
n\end{array} \frac{(n=60)}{n} \%$
\end{tabular}

Endoscopic severity

Mild (erythema, decreased vascular pattern, mild friability)

Moderate (marked erythema, absent vascular pattern, friability, erosion)

Severe (ulcers with spontaneous bleeding)

Quiescent

\begin{tabular}{rcrrrr}
5 & 8.6 & 4 & 7 & - & - \\
32 & 55.2 & 28 & 56 & - & - \\
21 & 36.2 & 18 & 36 & - & - \\
0 & - & 0 & - & 60 & 100 \\
\hline & & & & & \\
24 & 41.4 & 28 & 56 & 26 & 43.3 \\
18 & 31 & 14 & 28 & 20 & 33.3 \\
16 & 27.6 & 8 & 16 & 14 & 23.4
\end{tabular}

when compared to the patients with mild endoscopic changes (Table 4; Fig. 2b).

In what follows are the number of patients according to the endoscopic severity of UC: mild degree $(n=9)$ (erythema, mild friability) in endoscopy: 4 patients among the newly diagnosed patients, 5 patients in the relapse group; moderate degree $(n=60)$ (marked erythema, friability, erosions): 28 patients among the newly diagnosed patients, 32 patients in the relapse group; severe degree ( $n=39)$ (ulcers with spontaneous bleeding): 18 patients among the newly diagnosed patients, 21 patients in the relapse group; quiescent or normally appearing endoscopy in 60 patients with remission.

38 patients had lesions in the proctosigmoid region ( 8 in newly diagnosed patients, 16 in the relapse group, and
14 in remission group). Lesions in the left colon were found in 78 patients ( 28 in the newly diagnosed patients, 24 in the relapse group, and 26 in the remission group). Pancolitis was reported in 52 patients (14 in the newly diagnosed patients, 18 in the relapse group, and 20 in the remission group).

The clinical severity showed a statistically significant association with all of the postulated markers; the extent of UC had no statistically significant association with any marker. Endoscopic severity and histopathological activity showed statistically significant associations with all of the markers except the platelet count $(p=0.058,0.57)$ (Table 5).

Fecal LF showed a statistically significant association with $\operatorname{ESR} 1(p=0.000), \operatorname{CRP}(p=0.000), \operatorname{NLR}(p=0.000)$, 
Table 6. Correlation of ulcerative colitis markers with site of lesion, clinical severity, endoscopic severity, and histological activity

\begin{tabular}{cccll}
\hline Variables & Extent & $\begin{array}{l}\text { Clinical } \\
\text { severity }\end{array}$ & $\begin{array}{l}\text { Endoscopic } \\
\text { severity }\end{array}$ & $\begin{array}{l}\text { Pathological } \\
\text { activity }\end{array}$ \\
\hline Lactoferrin & & & & \\
$r$ & 0.2 & 0.8 & 0.81 & 0.5 \\
$p$ & 0.2 & $\mathbf{0 . 0 0 0}$ & $\mathbf{0 . 0 0 0}$ & $\mathbf{0 . 0 0 0}$ \\
ESR1 & & & & \\
$r$ & 0.1 & 0.71 & 0.56 & 0.32 \\
$p$ & 0.5 & $\mathbf{0 . 0 0 6}$ & $\mathbf{0 . 0 0 0}$ & $\mathbf{0 . 0 3}$ \\
CRP & & & & \\
$r$ & 0.13 & 0.76 & 0.7 & 0.53 \\
$p$ & 0.4 & $\mathbf{0 . 0 0 0}$ & $\mathbf{0 . 0 0 0}$ & $\mathbf{0 . 0 0 0}$ \\
NLR & & & & \\
$r$ & 0.19 & 0.61 & 0.68 & 0.4 \\
$p$ & 0.2 & $\mathbf{0 . 0 0 0}$ & $\mathbf{0 . 0 0 0}$ & $\mathbf{0 . 0 0 8}$ \\
PLT & & & & \\
$r$ & -0.14 & 0.36 & 0.29 & 0.09 \\
$p$ & 0.3 & $\mathbf{0 . 0 1}$ & 0.058 & 0.57 \\
MPV & & & & \\
$r$ & -0.18 & -0.45 & -0.42 & -0.43 \\
$p$ & 0.2 & $\mathbf{0 . 0 0 3}$ & $\mathbf{0 . 0 0 6}$ & $\mathbf{0 . 0 0 4}$ \\
\hline & & & &
\end{tabular}

Figures in bold are significant. $r$, correlation coefficient in Spearman's rank correlation.

Table 7. A noninvasive score for prediction of activity of ulcerative colitis

\begin{tabular}{lllll}
\hline Independent predictor & $\begin{array}{l}\text { Odd } \\
\text { ratio }\end{array}$ & $p$ & $\begin{array}{l}\text { Regression } \\
\text { coefficient }\end{array}$ & Score \\
\hline MPV $(<8.8 \mathrm{fL})$ & 15 & 0.004 & 2.7 & 1 \\
NLR $(>2.35)$ & 17 & 0.002 & 2.85 & 1 \\
ESR1 $(>29.5 \mathrm{~mm} / \mathrm{L})$ & 15 & 0.004 & 2.7 & 1 \\
Fecal lactoferrin & & & & \\
$\quad(>148.5 \mu \mathrm{g} / \mathrm{mL})$ & 10 & 0.006 & 2.3 & 1 \\
CRP $(>3.85 \mathrm{mg} / \mathrm{L})$ & 19.3 & 0.000 & 2.96 & 1 \\
Stool WBC $(>9 / \mathrm{HPF})$ & 24 & 0.001 & 3.1 & 1 \\
Stool RBC $(>6 / \mathrm{HPF})$ & 20 & 0.001 & 3 & 1 \\
\hline
\end{tabular}

platelet count $(p=0.01), \operatorname{MPV}(p=0.003)$, albumin $(p=$ $0.001)$, stool RBCs $(p=0.000)$, and stool WBCs $(p=$ $0.000)$.

The cutoff value of each variable significantly associated with activity in UC was determined using ROC curve analysis.

Fecal LF at a cutoff of $148.5 \mu \mathrm{g} / \mathrm{mL}$ has a sensitivity of $80 \%$ and a specificity of $72 \%$ in predicting activity in UC (area under the curve [AUC] 0.9, $p=0.001,95 \%$ CI 0.81-

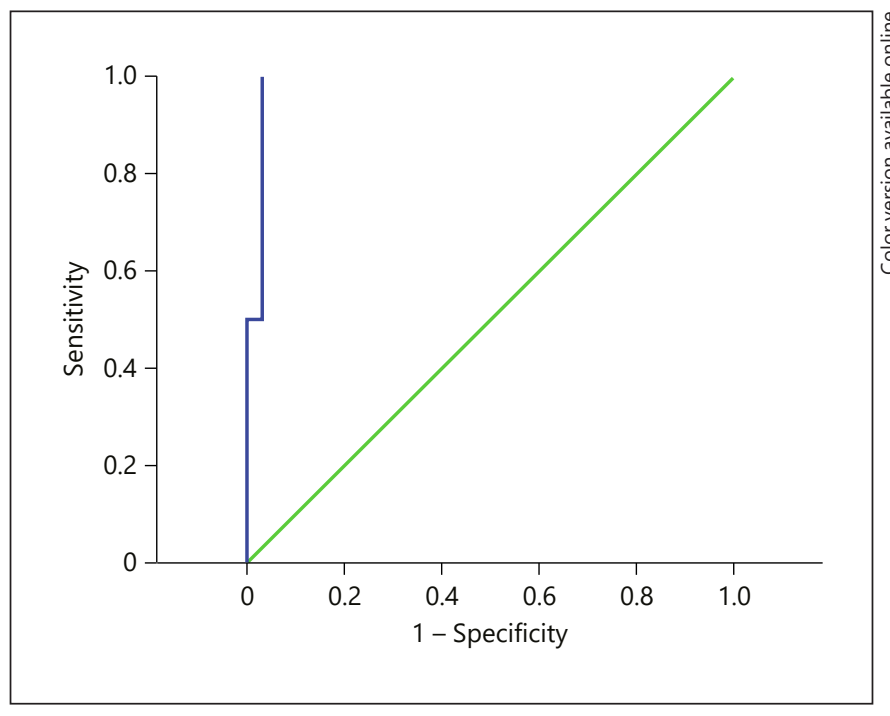

Fig. 3. ROC curve to estimate the predictive power of UC score in the study patients.

0.99). NLR at a cutoff of 2.35 has a sensitivity of $74 \%$ and a specificity of $86 \%$ in predicting activity in UC (AUC $0.81, p=0.01,95 \%$ CI $0.68-0.94$ ). ESR1 at a cutoff of 29.5 $\mathrm{mm} / \mathrm{h}$ has a sensitivity of $71 \%$ and a specificity of $86 \%$ in predicting activity in UC (AUC $0.79, p=0.03,95 \%$ CI $0.58-0.92)$. CRP at a cutoff of $6.85 \mathrm{mg} / \mathrm{L}$ has a sensitivity of $88 \%$ and a specificity of $71.7 \%$ in predicting activity in UC (AUC $0.890, p=0.001,95 \%$ CI $0.82-1$ ). MPV at a cutoff of $8.8 \mathrm{fL}$ has a sensitivity of $71 \%$ and a specificity of $86 \%$ in predicting activity in UC (AUC $0.837, p=0.005$, 95\% CI 0.69-0.97).

Fecal WBCs at a cutoff of 9 cells/HPF have a sensitivity of $80 \%$ and a specificity of $85.7 \%$ in predicting activity in UC (AUC 0.93, $p=0.001,95 \%$ CI 0.86-1). Fecal RBCs at a cutoff of 6 cells/HPF have a sensitivity of $77 \%$ and a specificity of $86 \%$ in predicting activity in UC (AUC $0.857, p=0.003,95 \%$ CI 0.74-0.96).

A proposed scoring system was postulated for the prediction of disease activity in patients with UC (Table 6). A score $\geq 5$ can detect $94 \%$ of cases of active UC as determined by the ROC curve with a sensitivity of $94 \%$ and a specificity of $100 \%$ (AUC 0.92 , SE $0.05, p=0.001,95 \%$ CI $0.82-1.1$ ) (Fig. 3). The application of the score in the study patients correlating it with clinical, endoscopic and histological severity is shown in Table 7.

\section{Validation Group}

The validation group included 60 patients ( 36 females, 24 males) with a mean age of $36.2 \pm 7.6$ years. The mean 
value of their activity score was $3.5 \pm 2.3$. 36 patients (60\%) showed a score $\leq 4$. According to Truelove and Witts' criteria, $24 / 36$ patients showed mild clinical manifestations (67\%), 12/36 patients (33\%) showed moderate manifestations, and their endoscopic severity according to the Mayo Clinic subscore revealed that $12 / 36$ patients were quiescent and had a healed mucosa (33\%), mild erythema was found in 18/36 (50\%) and moderate erythema in $6(17 \%)$, with a corresponding score of $3.5 \pm 0.6$.

Among patients with a score $>4(n=24,40 \%), 18$ patients $(75 \%)$ showed severe clinical manifestations and 6 patients (25\%) showed fulminant colitis; their endoscopic evaluation revealed moderate activity in 6 patients (25\%) and severe affection with ulceration in 18 (75\%); their corresponding score was $6 \pm 0.7$.

\section{Discussion}

Noninvasive markers of UC may have the potential advantage of avoiding invasive diagnostic tests and their complications [10]. Endoscopy can accurately judge the disease severity and assesses mucosal healing; however, it is invasive, contraindicated in severe or fulminant colitis, costly and has poor patient acceptability or acceptance.

The fecal biomarkers calprotectin and LF are neutrophil-derived proteins that are stable in feces and can be detected in small stool samples. Concentrations of both are raised in patients with gastrointestinal mucosal inflammation. They provide an inexpensive, noninvasive method of testing for active inflammation. They can be used for screening of IBD or mucosal healing and for monitoring the response to medical or surgical interventions. When compared to calprotectin, fecal LF had a higher sensitivity ( 80 vs. $78 \%$ ), specificity ( 85 vs. $83 \%$ ), PPV (87 vs. $86 \%$ ), and diagnostic accuracy (81 vs. $80 \%$ ) in predicting intestinal inflammation [11].

LF has a higher sensitivity and specificity during the diagnosis of suspected UC than is the case in Crohn's disease [12] and only LF at baseline was associated with a significantly higher relative risk of flare (RR 1.69; $p=$ 0.018 ), when compared to calprotectin, PMN, and CRP, which are also predictive of a flare [13]. Quantitative immunochemical fecal occult blood measurement is comparable to fecal LF and is easier and more useful than fecal calprotectin in predicting early relapse [14].

The cutoff value of $7.25 \mu \mathrm{g} / \mathrm{mL}$ for indicating elevated fecal LF had been established and validated for use in patients with IBD [15]. In the current study, fecal LF was significantly higher in newly diagnosed and relapse

Noninvasive Score in Ulcerative Colitis groups when compared to the remission group; a study by Borkowska et al. [16] found that the mean concentration in patients with active disease was 2,020 $\pm 1,076 \mu \mathrm{g} /$ $\mathrm{mL}$ and in patients with inactive disease $136 \pm 41 \mu \mathrm{g} / \mathrm{mL}$ $(p=0.001)$; interestingly during remission, LF level did not return to normal.

Fecal LF at a cutoff value of $148.5 \mu \mathrm{g} / \mathrm{ml}$ had a sensitivity of $80 \%$ and a specificity of $72 \%$ in predicting activity in ulcerative colitis, in comparison to a study which displayed that LF level $(>100 \mu \mathrm{g} / \mathrm{mL})$ showed a sensitivity of $87 \%$ and a specificity of $68 \%$ to identify clinically active $\mathrm{UC}[17]$.

Fecal LF correlated with the endoscopic severity being higher in moderate and severe cases and with histological activity proven by biopsy, which is in agreement with a study by Langhorst et al. [18] who had proven a significant correlation between endoscopic inflammation scores and fecal LF $(p<0.001)$.

Fecal LF was significantly higher in patients with UC than in healthy controls and correlated significantly with ESR, hematocrit, albumin, and platelet count $(p<0.001)$. Patients who experienced a clinical flare displayed higher fecal LF levels than those who remained in a clinical remission $[17,19]$. A study made by Masoodi et al. [20] observed that a significant fall in CRP, fecal myeloperoxidase, and fecal LF paralleled with the reduction in the Mayo Clinic subscores.

In the current study, total leukocyte count was only able to identify histological activity $(p=0.016)$. NLR at a cutoff value of 2.35 could discriminate between active and a quiescent UC. NLR was elevated compared to inactive UC patients and controls, and a cutoff value of 2.16 indicated the presence of active disease [21-23].

MPV was modulated by a series of cytokines activated in UC. It has an inverse relationship with the disease activity. MPV was correlated significantly with endoscopic severity (mild and severe) $(p=0.03)$ and histological activity $(p=0.01)$, which is supported by Kapsoritakis et al. [24].

We postulated an activity prediction score composed of highly correlated, easily calculated, reproducible, and practical variables; a score $>5$ could identify active UC with a sensitivity of $94 \%$ and a specificity of $100 \%$, which will help monitor the disease activity, modulate therapy, and avoid repetition of unnecessary colonoscopies. Also, the score was able to discriminate deep remission from clinical and endoscopic remissions. It was tested by its application on a validation group and was efficient in identifying severely affected patients with a sensitivity of $95 \%$ and a specificity of $85.7 \%$. The limitation of this study was 
being a single-center study that needed propagation in a wider scale. In conclusion, the activity score was postulated with high performance and should be applied in a wider scale of patients.

\section{Acknowledgements}

Special thanks go to the Clinical Pathology and Diagnostic Radiology Department physicians at the Zagazig University Hospital for their efforts and great help in this study.

\section{Statement of Ethics}

The study was conducted in accordance with the protocol and ethical principles of the university committee, and with the principles of the Declaration of Helsinki. Institutional review board approval of the study, informed consent forms, and patient information were provided.

\section{Disclosure Statement}

The authors declare that there is no conflict of interest.

\section{References}

1 Button LA, Roberts SE, Goldacre MJ, et al: Hospitalized prevalence and 5-year mortality for IBD: record linkage study. World J Gastroenterol 2010;16:431-438.

2 Angriman I, Scarpa M, D'Incà R, et al: Enzymes in feces: useful markers of chronic inflammatory bowel disease. Clin Chim Acta 2007;381:63-68.

3 Dai J, Liu WZ, Zhao YP, et al: Relationship between fecal lactoferrin and inflammatory bowel disease. Scand J Gastroenterol 2007;42: 1440-1444.

4 de la Motte DC, Fiocchi C: Platelets in inflammatory bowel disease: clinical, pathogenic, and therapeutic implications. Am J Gastroenterol 2004;99:938-945.

5 Yazici S, Yazici M, Erer B, et al: The platelet indices in patients with rheumatoid arthritis: mean platelet volume reflects disease activity. Platelets 2010;21:122-125.

6 Halazun KJ, Aldoori A, Malik HZ, et al: Elevated preoperative neutrophil to lymphocyte ratio predicts survival following hepatic resection for colorectal liver metastases. Eur J Surg Oncol 2008;34:55-60.

7 Truelove SC, Witts LJ: Cortisone in ulcerative colitis; final report on a therapeutic trial. $\mathrm{Br}$ Med J 1955;2:1041-1048.

8 Schroeder KW, Tremaine WJ, Ilstrup DM: Coated oral 5-aminosalicylic acid therapy for mildly to moderately active ulcerative colitis. A randomized study. N Engl J Med 1987;317: 1625-1629.

9 Sullivan LM, Massaro JM, Agostino BD: Presentation of multivariate data for clinical use: the Framingham Study risk score functions. Stat Med 2004;23:1631-1660.
10 Dubinsky MC, Ofman JJ, Urman M, et al: Clinical utility of serodiagnostic testing in suspected pediatric inflammatory bowel disease. Am J Gastroenterol 2001;96:758-765.

11 D’Incà R, Dal Pont E, Di Leo V, et al: Calprotectin and lactoferrin in the assessment of intestinal inflammation and organic disease. Int J Colorectal Dis 2007;22:429-437.

12 Wang Y, Pei F, Wang X, Sun Z, Hu C, Dou H: Diagnostic accuracy of fecal lactoferrin for inflammatory bowel disease: a meta-analysis. Intl J Clin Exp Pathol 2015;8:12319-12332.

13 Langhorst J, Boone J, Lauche R, Rueffer A, Dobos G: Faecal lactoferrin, calprotectin, PMN-elastase, CRP, and white blood cell count as indicators for mucosal healing and clinical course of disease in patients with mild to moderate ulcerative colitis: post hoc analysis of a prospective clinical trial. J Crohns Colitis 2016;10:786-794.

14 Kuriyama M, Kato J, Takemoto K, Hiraoka S, Okada H, Yamamoto K: Prediction of flareups of ulcerative colitis using quantitative immunochemical fecal occult blood test. World J Gastroenterol 2010;16:1110-1114.

15 Kane S: Inflammatory bowel disease in pregnancy. Gastroenterol Clin North Am 2003;32: 323-340.

16 Borkowska A, Liberek A, Łuczak G, et al: Fecal lactoferrin, a marker of intestinal inflammation in children with inflammatory bowel disease. Acta Biochim Pol 2015;62:541-545.

17 Walker TR, Land ML, Kartashov A, et al: Fecal lactoferrin is a sensitive and specific marker of disease activity in children and young adults with inflammatory bowel disease. J Pediatr Gastroenterol Nutr 2007;44:414-422.
18 Langhorst J, Elsenbruch S, Koelzer J, et al: Noninvasive markers in the assessment of intestinal inflammation in inflammatory bowel diseases: performance of fecal lactoferrin, calprotectin, and PMN-elastase, CRP, and clinical indices. Am J Gastroenterol 2008; 103: 162-169.

19 Kane SV, Sandborn WJ, Rufo PA, et al: Fecal lactoferrin is a sensitive and specific marker in identifying intestinal inflammation. Am J Gastroenterol 2003;98:1309-1314.

20 Masoodi I, Kochhar R, Dutta U, et al: Fecal lactoferrin, myeloperoxidase and serum Creactive are effective biomarkers in the assessment of disease activity and severity in patients with idiopathic ulcerative colitis. J Gastroenterol Hepatol 2009;24:1768-1774.

21 Torun S, Tunc BD, Suvak B, et al: Assessment of neutrophil-lymphocyte ratio in ulcerative colitis: a promising marker in predicting disease severity. Clin Res Hepatol Gastroenterol 2012;36:491-497.

22 Celikbilek M, Dogan S, Ozbakır O, et al: Neutrophil-lymphocyte ratio as a predictor of disease severity in ulcerative colitis. J Clin Lab Anal 2013;27:72-76.

23 Demira K, Demirtasa A, Kayaa SU, et al: The relationship between the neutrophil-lymphocyte ratio and disease activity in patients with ulcerative colitis. Kaohsiung J Med Sci 2015; 31:585-590.

24 Kapsoritakis AN, Koukourakis MI, Sfiridaki A, et al: Mean platelet volume: a useful marker of inflammatory bowel disease activity. Am J Gastroenterol 2001;96:776-781. 